largely restricted to the pre-central convolution. It is well known that in embolism we sometimes have an effect beyond the locality of the obstructive lesion, so that the post- and pre-central convolutions may have both been involved in the acute condition of the lesion. The impairment of sensation in such cases from purely motor lesion, may be due to disruption of the neuronal associations between the sensory and the motor area.

Dr. A. A. Eshner said he wondered whether it was perfectly precise to designate this case as one of monoplegia. Although Dr. Carncross demonstrated that the paralysis predominates in one upper extremity, he also pointed out that the reflexes in the lower extremities are exaggerated, which must indicate some involvement of the motor fibers passing to these parts. With respect to the situation of the lesion, the question naturally arises whether in the presence of a cortical lesion one would not have a right to expect some convulsive tendency, either a convulsion at the inception of the disease, or epileptic seizures since them.

Dr. Spiller said there are a few cases of monoplegia from lesion of the internal capsule on record. The case presented by Dr. Carncross, he thought, must be regarded as cortical in origin. The middle cercbral supplies the whole of the motor cortex of the brain except the extreme upper part. Dr. Spiller has a brain in which there was complete destruction of the middle cerebral and of the whole Sylvian distribution, yet the uppermost part of the pre-central convolution and the whole of the para-central lobule were preserved, as that portion of the brain is nourished from the anterior cerebral artery. In that case the fibers coming from the para-central lobule were destroyed within the white matter of the brain by the sclerotic lesion and there was hemiplegia. Dr. Spiller had another brain in which there is destruction of the cortex in the central convolutions only in the upper part, in the center for the tpper limb. In the case presented by Dr. Carncross, we may suppose there was an embolus of the terminal portion of the middle cerebral artery, and that the cortex of the upper limb center was destroyed; it is possible that the anterior cerebral artery extended further downward on the lateral surface of the brain than in most persons, and that the center for the lower limb has been chiefly supplied by this artery.

Dr. Spiller doubted whether anyone can find in the literature a case of cerebral monoplegia of the upper limb without some involvement of the face. Monoplegia is to this extent a misnomer. $\mathrm{He}^{\circ}$ did not observe any weakness of the orbicularis palpebrarum muscle or of the flexors in the lower limb in this case that Dr. Carncross had shown.

Dr. Carncross did not know whether convulsive seizures had ever occurred. The increased reflexes are in the right and left leg and right arm, and especially in the left arm.

\title{
A CASE OF UNILATERAL HEMORRHAGE IN THE MEDULLA FOLLOWED BY SYRINGOMYELIC SENSORY DISTURBANCES.
}

By Alfred Gordon, M.D.

A man of 48 was suddenly seized with an intense vertigo, objects turning to the right. He soon lost consciousness. An hour later there was inability to swallow and expectorate, also shortness of breath. Upon examination were found right facial palsy, narrowness of the right palpebral fissure, with marked tremor of the tongue, difficulty in moving the 
tongue, complete loss of the three fornss of sensation on the entire left side, cerebellar asynergy, holding the head on the right shoulder, and titubation with a tendency to walk to the right.

At present he has some difficulty in swallowing on the right side, some shortness of breath, some facial deviation to the left, narrowness of the right palpebral fissure, syringomyelic sensory dissociation on the left side including the face, tremor and atrophy of tongue with R.D., and stiffness of the jaws.

Dr. Gordon said, as to the possibility of polioencephalitis, he considered that at the beginning, but he was absolutely unable to explain the condition in that way. The cerebellar attitude of the head, and the cerebellar gait were most typical of a cerebellar or peduncular involvement. How polioencephalitis could affect at the same time fibers of the peduncle he could not explain, so he had diagnosed hemorrhage. The latter could explain all the symptoms. The cerebellar symptoms cleared up.

As far as he knows only a few fibres of Gowers' tract enter the white reticulated substance. The majority of the fibers of Gowers' tract go around the lateral lemniscus to the superior cerebellar peduncle. Assuming that the fibers of the white reticulated substance are involved on one side, the minority of Gowers' tract would also be affected. That the destruction of a few fibres of Gowers' tract would give such a complete picture of sensory disassociation is a question which has remained unsolved in Dr. Gordon's mind. When he examined the patient three days after the apoplectic insult he found while pain and temperature were entirely abolished on the left side, touch was also somewhat affected, but later touch returned. As far as the sensory disturbance of the face is concerned, of course Dr. Dercum's suggestion would perhaps explain the question of polioencephalitis. But on the other hand it does go hand in hand with the entire picture.

\section{THREE CASES OF LESION OF THE EPICONUS.}

By William G. Spiller, M.D.

Minor, of Moscow, has pointed out that a lesion of the gray matter of the fifth lumbar and first and second sacral segments of the spinal cord gives a definite clinical picture, characterized by the presence of certain symptoms and the absence of others seen in lesions of the conus. $\mathrm{He}$ has given the name of epiconus to the portion of the cord mentioned. The symptoms are atrophy and paralysis of motion and sensation in the innervation of the sacral plexus, especially in that of the peroneal nerves. The gait is of the steppage type because of foot-drop. The flexors on the back of the thighs and gluteal muscles may be weak because of the implication of the fifth lumbar segment. The Achilles tendon reflexes and plantar reflexes are lost. The sphincters of bladder and rectum and the sexual functions are not affected because the conus in which the centers for these functions and muscles are situated and the white columns above the conus are not implicated. The patellar reflexes are preserved, as the lesion does not extend into the fourth lumbar segment.

Dr. Spiller has had three cases of lesion of the epiconus. The first and third were traumatic in origin. The first has already been reported by Dr. Weisenburg.

The second case was seen with Dr. Alfred Stengel. The symptoms developed after pneumonia, and the diagnosis was poliomyelitis of the 